\title{
Sistema de informação como ferramenta de cálculo e gestão de custos em laboratórios de análises clínicas
}

\section{Laboratory information system in the assessment and management of costs in clinical laboratories}

Katia Cristina Ugolini Mugnol'; Marcos Bosi Ferraz²

\begin{abstract}
unitermos
Sistemas de informação em

laboratório clínico

Custos e análises de custos

Economia da saúde

Informática em saúde

\section{resumo}

O laboratório clínico, munido de um sistema de informática que costumeiramente operacionaliza suas rotinas e atua como um importante arquivo de dados e informações, pode utilizar-se deste como uma ferramenta que seria de grande utilidade para o cálculo e a gestão do custo real dos exames e demais procedimentos que realiza. Os sistemas implementados no momento, entretanto, segundo os dados obtidos junto a nove laboratórios situados na cidade de São Paulo, são tidos por seus usuários como inadequados a esta finalidade. As opiniões a respeito foram as mesmas, independentemente do tempo em que o sistema encontra-se em operação, de sua procedência, do porte do laboratório, do tipo de atendimento que pratica, de sua situação econômico-financeira, de seu grau de automação, da formação profissional dos gestores e dos recursos que têm disponíveis.
\end{abstract}

\section{abstract}

The computer system generally used in clinical laboratories in the acomplishment of their routines, as an important archive of data and information, can also be used as a tool that would be of great utility for the assessment and the management of the real cost of exams and other procedures. The systems implemented at the moment, however, according to nine laboratories of the city of São Paulo, are considered by its users as inadequate to this purpose. The opinions were the same independently of the system operation time, its origin, type of attendance, economic situation, degree of automation, professional formation of managers or of available resources. key words

Laboratory information

system

Costs and costs assessment

Health economics

Health computer science

\section{Introdução}

O laboratório clínico é uma instituição que vem passando por uma infinidade de modificações nas últimas décadas, seja para acompanhar a evolução tecnológica marcante do século $X X$, seja para firmar-se num mercado cada vez mais exigente, mais competitivo. A evolução da ciência médica trouxe consigo a necessidade de métodos diagnósticos cada vez mais sensíveis, mais precisos e que trazem consigo quase sempre um maior custo. Paralelamente, como muitas outras instituições de saúde, o laboratório tem sofrido impactos profundos em função das mudanças no quadro econômico e político de nosso país. Passou a sofrer as pressões do mercado e o estresse das oscilações financeiras, o que vem desafiando a todos na busca por um aprendizado a curto prazo dos fundamentos da gestão de custos.

1. Biomédica; mestra profissional em Economia da Saúde pela Universidade Federal de São Paulo (UNIFESP); mestra e doutoranda em Biotecnologia pela Universidade de Mogi das Cruzes (UMC). 2. Médico; doutor em Reumatologia pela UNIFESP; pós-doutor em Economia da Saúde pela McMaster University (Canadá); vice-presidente do Conselho de Administração do Fleury - Medicina Diagnóstica.

Trabalho baseado em tese intitulada Aplicação dos Sistemas de Informática no Cálculo e na Cestão de Custos em Laboratórios de Análises Clínicas apresentada na UNIFESP em 2003 para obtenção do título de mestra profissional em Economia da Saúde por Katia Cristina Ugolini Mugnol sob a orientação do Prof. Dr. Marcos Bosi Ferraz.

Trabalho apresentado no 370 Congresso Brasileiro de Patologia Clínica e Medicina Laboratorial, realizado no Rio de Janeiro de 24 a 27 de setembro de 2003, e no IX Congresso Brasileiro de Biomedicina, realizado em São Paulo de 21 a 24 de abril de 2004. 
Da descoberta da célula ao desenvolvimento das técnicas de DNA recombinante, muito aconteceu para tornar o laboratório clínico um centro de apoio diagnóstico e de tratamento de grande importância. Modernas técnicas e tecnologias foram e continuam sendo implementadas, fazendo deste universo uma realidade dinâmica. Conseqüentemente, a necessidade de adaptação às novas exigências também passou a ser uma constante, tanto nos aspectos técnicos quanto administrativo-comerciais. Tornou-se necessário, acima de tudo, incorporar à visão exclusivamente técnica os conceitos administrativos e gerenciais, pois o laboratório deixou de ser um anexo diagnóstico dos serviços de saúde e passou a existir como uma empresa real, com as responsabilidades e necessidades inerentes a tal estrutura. Seus administradores, até então de formação voltada quase sempre de forma exclusiva às áreas médicas, passaram a buscar o aprimoramento necessário à absorção das novas funções, deixando de ser puramente técnicos para tornarem-se também gestores. Sem competência gerencial, principalmente em face de mutações que permeiam a macroeconomia globalizada, os administradores laboratoriais estarão à mercê do status quo da insegurança, do descontrole e, como conseqüência, bem perto do fracasso econômico ${ }^{(1)}$. Tornaram-se caçadores ansiosos por dados e informações gerenciais e por ferramentas capazes de fornecer subsídios para a tomada de decisões com maior índice de aceite e segurança.

Entre as ferramentas gerenciais, a informatização de sistemas e processos aparece em destaque e, como advento do século XXI, entra em cena a tecnologia da informação, que foi citada por Friedman no universo laboratorial como sendo uma tecnologia "que permite criar uma nova economia e novos modelos administrativos".

No final dos anos 1950, o computador já aparecia nos laboratórios de análises clínicas incorporado aos equipamentos, visando a economia de tempo na execução dos processos analíticos, favorecendo análises mais homogêneas, menos sujeitas às variáveis decorrentes da intervenção humana, e trazendo consigo a esperança da redução de custos, principalmente com mão-de-obra. Aos poucos, a informática passou também a fazer parte dos processos operacionais e de apoio ao laboratório como um todo, da recepção do cliente à entrega dos resultados, tornando-se uma forma inteligente de armazenamento, consulta e administração de dados. Hoje surge incorporada a conceitos de eficiência, eficácia, agilidade e qualidade.

Por volta de 1960 surgiu o conceito de laboratory information system (LIS), traduzido como sistema de informática laboratorial e descrito como sendo um sistema composto por um ou mais softwares, ligados direta ou indiretamente, que visam gerenciar informações dentro de um laboratório de análises clínicas, sejam estas de cunho técnico, operacional, administrativo, gerencial ou de uma mescla destes. Deve ser entendido como uma entidade dissociada dos sistemas de automação laboratorial, com quem pode ter uma relação de grande intimidade, quase simbiótica, mas de quem deve ser desvinculado quando se quer compreender sua real finalidade. A automação laboratorial, por sua vez, pode ser visualizada como parte do laboratory automation system (LAS), um conjunto que engloba as atividades de gerenciamento de processos envolvidos no controle de equipamentos e instrumentos laboratoriais, controle de amostras e processos analíticos (Blick, 1997) $)^{(6,8)}$.

Automação e informatização, portanto, são fatores que por si só alteraram o ambiente laboratorial, tornando-o mais produtivo, mais eficiente, mais controlado. Cresce também a necessidade de empregá-los para um maior conhecimento dos custos envolvidos no processo produtivo, mais pontualmente, do custo dos exames.

O cálculo do custo real de um exame laboratorial é bastante complexo, havendo uma gama enorme de variáveis decorrentes do tipo de atividade desenvolvida, da estrutura em que o laboratório está instalado, do tipo e do grau de complexidade dos exames que realiza, do tipo de cliente atendido, do perfil dos profissionais que lá trabalham ou se utilizam dos serviços, do caráter técnico assumido pelo serviço, do nível de automação presente, das regras estabelecidas pela legislação vigente e assim por diante. São custos diretos e indiretos, fixos e variáveis, todos influenciando o custo final de um procedimento. Pensando apenas na fase analítica, por exemplo, é preciso considerar o nível de repetições necessárias à liberação de um resultado, os testes consumidos para calibração do equipamento e para o controle do analito, as perdas inerentes à própria metodologia empregada e às possíveis falhas dos instrumentos empregados, o custo da mão-de-obra envolvida em todas as fases do processo e dos demais insumos utilizados e que não estão diretamente ligados à análise em si. Têm-se ainda as perdas inerentes à pouca estabilidade ou curta validade de alguns reagentes, que são descartados sem que sejam utilizados em sua totalidade; os custos adicionais vinculados à urgência na realização de alguns testes, o que quebra as regras estabelecidas para o melhor aproveitamento dos insumos; as perdas resultantes da depreciação dos equipamentos e da baixa demanda em determinados períodos do ano; os custos com a manutenção preventiva 
e corretiva destes equipamentos, com o transporte das amostras até o laboratório, com os atendimentos diretos e assim por diante.

Todas essas variáveis, por sua vez, não são uma constante absoluta entre os laboratórios, sendo particulares a cada um deles conforme suas características individuais, o que torna necessário que as ferramentas utilizadas para o cálculo do custo sejam capazes de distinguir as nuances de cada face técnico-administrativa. A fidelidade deste cálculo é capaz de fundamentar a tomada mais segura de decisões simples, como a modificação de rotinas de trabalho e a terceirização de análises, até decisões de maior grau de complexidade, como a aquisição de novos equipamentos e até da mudança do perfil da empresa. A gestão dos custos é o que proporciona ao laboratório a oportunidade de promover de forma eficiente e efetiva a utilização dos recursos disponíveis, os quais costumam andar sempre atrás da evolução tecnológica, o que reforça ainda mais a necessidade de ferramentas que permitam o seu aproveitamento ao máximo. É preciso avaliar de forma simultânea a produtividade, a qualidade e os custos, não sendo mais aceitável que seja dada prioridade apenas aos dois primeiros sem levar o terceiro em conta.

O LIS, associado ao próprio LAS, pode ter uma nobre aplicação na difícil tarefa de calcular o custo real dos exames, sendo uma ferramenta de extrema eficiência como fonte de informações consolidadas para a tomada de decisões gerenciais dos mais diferentes níveis. $\mathrm{O}$ software utilizado deve permitir planejamento e otimização de custos por análises simuladas, por séries de segmento de custo e por comparação entre alternativas. A base de dados essencial para a administração do custo deve, portanto, consistir em informações detalhadas de custos diretos e indiretos e de toda informação relevante ${ }^{(7)}$.

Tendo-se estes argumentos em mente, o objetivo primário deste trabalho foi demonstrar a situação atual dos sistemas de informática laboratorial como sendo este tipo de ferramenta capaz de fornecer subsídios para o cálculo e a gestão dos custos dos exames realizados por laboratórios de análises clínicas.

\section{Metodologia}

Foram pesquisados nove laboratórios situados na cidade de São Paulo, selecionados de modo aleatório, por sorteio, entre uma população de 224 laboratórios de análises clínicas, estes submetidos inicialmente a um processo de estratificação em três grandes grupos conforme a média mensal de exames que realizam (Tabela 1). A população foi constituída através da relação de laboratórios disponibilizada pela OESP Mídia e a estratificação, fundamentada em impressões pessoais e de mercado e confirmada posteriormente durante a pesquisa junto aos participantes selecionados. Foram desconsiderados para composição desta população os laboratórios que atuam com outras especialidades que não as análises clínicas ou que atuam como fornecedores de insumos para estes, bem como os que se constituem apenas em postos de coleta de amostras. Os que se caracterizam como centros de medicina diagnóstica tiveram considerados na pesquisa apenas os dados e as informações relacionados a atividades como laboratório de análises clínicas, incluindo-se aqui a anatomia patológica e a citologia oncótica.

O projeto previa a participação de 18 laboratórios, mas devido ao baixo índice de adesão à pesquisa a amostragem foi reduzida à metade. Foi necessário o sorteio de 67 laboratórios para que se conseguisse um total de nove participantes, o que representou $86,6 \%$ de recusa de participação neste estudo. Foram necessários cinco ciclos de sorteio para a composição de uma amostra em que o número de participantes de cada um dos três grupos fosse 0 mesmo. A cada ciclo verificou-se o interesse do selecionado em participar do estudo, repassando-se a ele os objetivos e definindo-se, através de termo de consentimento livre e esclarecido, ser esta adesão voluntária e não-remunerada. Em caso de recusa em participar, independentemente dos motivos alegados, outro foi sorteado em seu lugar em um novo ciclo (Tabela 2).

Utilizou-se como instrumento de pesquisa um questionário elaborado especificamente para atender aos objetivos do trabalho, o qual foi aplicado em entrevista direta com os representantes dos laboratórios selecionados, acompanhado do termo de consentimento livre e esclarecido. Foram

\section{Tabela 1 Distribuição dos laboratórios listados}

\begin{tabular}{lcc} 
Grupos & Número de laboratórios & Porcentagem total \\
Grupo A & 18 & 8 \\
Grupo B & 43 & 19,2 \\
Grupo C & 163 & 72,8 \\
Total & 224 & 100 \\
\hline
\end{tabular}

Grupo A: laboratórios que realizam um total de exames, no período de um mês, igual ou superior a 50 mil; grupo B: laboratórios que realizam um total de exames, no período de um mês, superior ou igual a 20 mil e inferior a 50 mil; grupo C: laboratórios que realizam um total de exames, no período de um mês, inferior a 20 mil. A população laboratorial referida contempla os laboratórios presentes na cidade de São Paulo e foi composta com base em listagem disponibilizada pela OESP Mídia. 


\section{Tabela 2 Ciclo de sorteios para composição da amostra}

\begin{tabular}{lcccc}
\hline & Grupo A & Grupo B & Grupo C & Porcentagem de adesão por ciclo \\
Laboratórios sorteados - Ciclo 1 & 6 & 6 & 6 & 11.1 \\
Laboratórios que aderiram & 1 & 0 & 1 & 25 \\
Laboratórios sorteados - Ciclo 2 & 5 & 6 & 5 & \\
Laboratórios que aderiram & 1 & 2 & 1 & 8,3 \\
Laboratórios sorteados - Ciclo 3 & 4 & 4 & 4 & 9,1 \\
Laboratórios que aderiram & 1 & 0 & 0 & 10 \\
Laboratórios sorteados - Ciclo 4 & 3 & 4 & 4 & 67 \\
Laboratórios que aderiram & 0 & 1 & 0 & 9 \\
Laboratórios sorteados - Ciclo 5 & 3 & 3 & 4 & - \\
Laboratórios que aderiram & 0 & 0 & 1 & 13,4 \\
Total de laboratórios sorteados & 21 & 23 & 23 & \\
Total de adesões & 3 & 3 & 3 & \\
Porcentagem de adesão por grupo & 14,3 & 13 & 13 & - \\
Porcentagem de adesão geral & - & - & - & \\
\hline
\end{tabular}

considerados qualificados para o estudo os profissionais de nível hierárquico definidos como de nível I (diretoria/gerência) e de nível II (supervisão/coordenação), desde que participantes, na empresa, das decisões de ordem administrativa relevantes ao estudo. Dos nove laboratórios foram entrevistados 11 colaboradores que neles atuam em diversas funções, sendo $46 \%$ deles supervisores técnicos, $27 \%$ diretores administrativos, $9 \%$ coordenadores de informática, $9 \%$ supervisores de informática e $9 \%$ diretores científicos.

Tanto o questionário quanto os documentos a ele anexados foram identificados para permitir seu rastreamento, mas tanto a identidade do laboratório quanto dos entrevistados, bem como os dados gerenciais fornecidos, serão mantidos em sigilo permanente.

\section{Resultados}

Com base nas informações coletadas na entrevista através do preenchimento do questionário, foi montado o perfil básico dos laboratórios participantes (Tabela 3).

Com base nas respostas obtidas através do questionário e de outros dados gentilmente fornecidos pelos entrevistados, foram tabuladas as informações inerentes à relação entre os sistemas de informática laboratorial implementados e a gestão do custo dos exames, conforme demonstrado na Tabela 4. Vale ressaltar que, para todos os efeitos, foram considerados LIS apenas os softwares utilizados para a operacionalização das atividades realizadas nos laboratórios participantes e para o gerenciamento das informações a elas relacionadas, sendo desconsiderados aqueles que se apresentam como parte integrante dos equipamentos analíticos, seguindo-se os mesmos conceitos anteriormente estabelecidos.

Visando demonstrar o grau de satisfação dos laboratórios pesquisados quanto a rotinas específicas, foi-lhes solicitado que pontuassem o sistema que possuem para algumas delas. Foram atribuídos às rotinas conceitos na forma de notas de zero a cinco, em uma escala crescente de satisfação. Os conceitos aplicados foram submetidos ao cálculo de média ponderada e estão demonstrados na Tabela 5. O conceito máximo que pode ser alcançado por uma dada rotina é representado por um total de 45 pontos, que, após a média ponderada, totaliza um grau máximo de satisfação igual a cinco.

\section{Discussão}

Foi possível constatar, no decorrer do estudo, o foco predominantemente operacional do LIS implementado nos laboratórios pesquisados, tenham sido eles desenvolvidos por uma equipe pertencente à própria instituição ou adquiridos de terceiros. As rotinas de recepção e dos setores técnicos apareceram adequadamente sustentadas pelos sistemas de informática, mas, em contrapartida, as atividades relacionadas ao gerenciamento técnico-administrativo, ao controle de estoque e compras, ao acompanhamento dos programas de controle de qualidade e aos estudos de viabilidade e produtividade apareceram totalmente desprovidas de apoio por parte destes. 
Tabela 3 Características gerais dos laboratórios participantes

\begin{tabular}{|c|c|c|c|c|c|c|c|c|}
\hline & \multicolumn{2}{|c|}{ Grupo A } & \multicolumn{2}{|c|}{ Grupo B } & \multicolumn{2}{|c|}{ Grupo C } & \multicolumn{2}{|c|}{ Totais } \\
\hline & N & $\%$ & $\mathbb{N}$ & $\%$ & N & $\%$ & NT & $\% \mathrm{~T}$ \\
\hline \multicolumn{9}{|l|}{ Tipo de laboratório } \\
\hline Público & 0 & 0 & 1 & 33,3 & 0 & 0 & 1 & 11,1 \\
\hline Privado & 3 & 100 & 2 & 66,7 & 3 & 100 & 8 & 88,9 \\
\hline \multicolumn{9}{|l|}{ Quanto ao tipo de atendimento } \\
\hline Hospitalar & 0 & 0 & 1 & 33,3 & 0 & 0 & 1 & 11,1 \\
\hline Ambulatorial & 2 & 66,7 & 1 & 33,3 & 3 & 100 & 6 & 66,7 \\
\hline Misto & 1 & 33,3 & 1 & 33,3 & 0 & 0 & 2 & 22,2 \\
\hline \multicolumn{9}{|c|}{ Quanto aos setores técnicos atuantes } \\
\hline Anatomia patológica & 3 & 100 & 2 & 66,7 & 2 & 66,7 & 7 & 77,8 \\
\hline Biologia molecular & 1 & 33,3 & 1 & 33,3 & 0 & 0 & 2 & 22,2 \\
\hline Bioquímica & 3 & 100 & 3 & 100 & 3 & 100 & 9 & 100 \\
\hline Citologia & 3 & 100 & 2 & 66,7 & 2 & 66,7 & 7 & 77,8 \\
\hline Endocrinologia & 3 & 100 & 3 & 100 & 3 & 100 & 9 & 100 \\
\hline Hematologia & 3 & 100 & 3 & 100 & 3 & 100 & 9 & 100 \\
\hline Imunologia & 3 & 100 & 3 & 100 & 3 & 100 & 9 & 100 \\
\hline Liquor & 1 & 33,3 & 1 & 33,3 & 0 & 0 & 2 & 22,2 \\
\hline Microbiologia & 3 & 100 & 3 & 100 & 3 & 100 & 9 & 100 \\
\hline Parasitologia & 3 & 100 & 3 & 100 & 3 & 100 & 9 & 100 \\
\hline Urinálise & 3 & 100 & 3 & 100 & 3 & 100 & 9 & 100 \\
\hline Terceirizam análises & 3 & 100 & 3 & 100 & 3 & 100 & 9 & 100 \\
\hline \multicolumn{9}{|c|}{ Formação dos profissionais de nivel gerencial* } \\
\hline Médicos & \multicolumn{2}{|c|}{$40 \%$} & \multicolumn{2}{|c|}{$52 \%$} & \multicolumn{2}{|c|}{$38 \%$} & \multicolumn{2}{|c|}{$43 \%$} \\
\hline Biomédicos & \multicolumn{2}{|c|}{$12 \%$} & \multicolumn{2}{|c|}{$14 \%$} & \multicolumn{2}{|c|}{$21 \%$} & \multicolumn{2}{|c|}{$16 \%$} \\
\hline Bioquímicos & \multicolumn{2}{|c|}{$18 \%$} & \multicolumn{2}{|c|}{$17 \%$} & \multicolumn{2}{|c|}{$30 \%$} & \multicolumn{2}{|c|}{$22 \%$} \\
\hline Administradores & \multicolumn{2}{|c|}{$30 \%$} & \multicolumn{2}{|c|}{$17 \%$} & \multicolumn{2}{|c|}{$11 \%$} & \multicolumn{2}{|c|}{$19 \%$} \\
\hline Média mensal de exames & \multicolumn{2}{|c|}{95.000} & \multicolumn{2}{|c|}{34.000} & \multicolumn{2}{|c|}{13.000} & \multicolumn{2}{|c|}{-} \\
\hline Média do número de funcionários & \multicolumn{2}{|c|}{230} & & & & & & \\
\hline Média de unidades de coleta & & & & & & & & \\
\hline
\end{tabular}

$N$ = número de laboratórios, de cada grupo, que fazem parte do item indicado; \% = porcentagem de laboratórios que fazem parte do item indicado em relação ao total de participantes do grupo; NT = número total de laboratórios que fazem parte do item indicado; \%T = porcentagem de NT em relação ao total de laboratórios que participaram da pesquisa; *foram considerados os profissionais que desempenham funções em nível de diretoria e gerência.

Independentemente do porte do laboratório, de sua situação econômico-financeira, do tipo de atendimento, do grau de automação, da formação profissional de seus gestores e dos recursos que têm disponíveis, todos os entrevistados demonstraram opiniões semelhantes quanto ao LIS com que trabalham. Todos foram unânimes nas manifestações de insatisfação quanto à incapacidade do LIS como ferramenta gerencial, visto que não possuem modelos adequados ao cálculo e à gestão do custo dos exames, fornecendo apenas relatórios estatísticos que, apesar de serem úteis para muitas finalidades, não são capazes de embasar decisões que requeiram informações mais complexas. Torna-se assim difícil encontrar formas para a demonstração, a administração e a incorporação dos custos diretos e indiretos ao custo do exame em si.

Conforme as informações que constam nas Tabelas 3 e 4, pode-se perceber que todos os laboratórios partici- 


\begin{tabular}{|c|c|c|c|c|c|c|c|c|}
\hline & \multicolumn{2}{|c|}{ Grupo A } & \multicolumn{2}{|c|}{ Grupo B } & \multicolumn{2}{|c|}{ Grupo C } & \multicolumn{2}{|c|}{ Totais } \\
\hline & NS & $\%$ & NS & $\%$ & NS & $\%$ & NST & $\% \mathrm{~T}$ \\
\hline $\begin{array}{l}\text { Laboratórios participantes do estudo que possuem algum tipo } \\
\text { de metodologia para cálculo do custo de seus exames }\end{array}$ & 3 & 100 & 3 & 100 & 3 & 100 & 9 & 100 \\
\hline Fazem uso do LIS para esses cálculos & 3 & 100 & 3 & 100 & 3 & 100 & 9 & 100 \\
\hline LIS oferece estatísticas para gestão dos serviços & 3 & 100 & 3 & 100 & 3 & 100 & 9 & 100 \\
\hline $\begin{array}{l}\text { LIS oferece outros relatórios de gerenciamento além das } \\
\text { estatísticas }\end{array}$ & 1 & 33,3 & 0 & 0 & 0 & 0 & 1 & 11,1 \\
\hline $\begin{array}{l}\text { LIS oferece recursos para agregação dos custos diretos e } \\
\text { indiretos ao custo total do exame }\end{array}$ & 0 & 0 & 0 & 0 & 0 & 0 & 0 & 0 \\
\hline $\begin{array}{l}\text { LIS oferece recursos para agregação do custo com pessoal ao } \\
\text { custo total do exame }\end{array}$ & 0 & 0 & 0 & 0 & 0 & 0 & 0 & 0 \\
\hline $\begin{array}{l}\text { LIS oferece recursos para agregação do custo com serviços de } \\
\text { manutenção ao custo total do exame }\end{array}$ & 0 & 0 & 0 & 0 & 0 & 0 & 0 & 0 \\
\hline $\begin{array}{l}\text { LIS oferece recursos para agregação do valor relativo à } \\
\text { depreciação de equipamentos ao custo total do exame }\end{array}$ & 0 & 0 & 0 & 0 & 0 & 0 & 0 & 0 \\
\hline $\begin{array}{l}\text { LIS oferece recursos para relacionar o consumo de reagentes e } \\
\text { demais insumos ao número de exames realizados }\end{array}$ & 1 & 33,3 & 0 & 0 & 0 & 0 & 1 & 11,1 \\
\hline $\begin{array}{l}\text { LIS oferece recursos para incorporar as perdas relativas ao uso } \\
\text { de calibradores e controles ao custo total dos exames }\end{array}$ & 0 & 0 & 0 & 0 & 0 & 0 & 0 & 0 \\
\hline $\begin{array}{l}\text { LIS permite avaliar o custo adicional de exames solicitados em } \\
\text { caráter de urgência }\end{array}$ & 0 & 0 & 0 & 0 & 0 & 0 & 0 & 0 \\
\hline $\begin{array}{l}\text { LIS oferece recursos para incorporar ao custo total do exame } \\
\text { os gastos com insumos, medicamentos, produtos utilizados no } \\
\text { atendimento ao cliente }\end{array}$ & 0 & 0 & 0 & 0 & 0 & 0 & 0 & 0 \\
\hline LIS permite a gestão de gastos com laboratórios terceirizados & 1 & 33,3 & 0 & 0 & 0 & 0 & 1 & 11,1 \\
\hline LIS permite calcular a produtividade dos setores técnicos & 0 & 0 & 0 & 0 & 0 & 0 & 0 & 0 \\
\hline $\begin{array}{l}\text { LIS permite avaliar/simular custos utilizando metodologias } \\
\text { analíticas alternativas na realização de exames }\end{array}$ & 0 & 0 & 0 & 0 & 0 & 0 & 0 & 0 \\
\hline $\begin{array}{l}\text { LIS fornece dados para gestão de rotinas analíticas visando a } \\
\text { otimização de gastos e controle de custos }\end{array}$ & 0 & 0 & 0 & 0 & 0 & 0 & 0 & 0 \\
\hline $\begin{array}{l}\text { LIS permite acompanhar a evolução dos custos em tempo hábil } \\
\text { para a tomada de decisões }\end{array}$ & 0 & 0 & 0 & 0 & 0 & 0 & 0 & 0 \\
\hline
\end{tabular}

NS = número de laboratórios, de cada grupo, que responderam sim ao questionamento; \% = porcentagem de NS em relação ao total de participantes do grupo; NST = número total de laboratórios que responderam sim ao questionamento; \%T = porcentagem de NST em relação ao total de laboratórios que participaram da pesquisa.

pantes possuem algum tipo de metodologia para o cálculo do custo dos seus exames e se utilizam das estatísticas fornecidas por seus sistemas para isso. Entretanto, oito dos nove laboratórios não possuem qualquer outro tipo de relatório gerencial emitido pelo LIS além dessas estatísticas, e mesmo o que possui tal ferramenta demonstra insatisfação quanto à qualidade das informações fornecidas por este relatório, visto que todos os pesquisados atribuíram conceitos negativos a esta rotina na pesquisa do grau de satisfação.

Em 100\% dos pesquisados o LIS apresentou ausência de recursos que permitam agregar custos diretos e indiretos ao cálculo do custo dos exames. Nenhum deles permite computar, por exemplo, o custo com pessoal, serviços de manutenção, depreciação dos equipamentos, perdas relativas à aplicação das sistemáticas de controle de qualidade 


\begin{tabular}{|c|c|c|c|c|c|c|c|c|c|c|c|c|c|c|}
\hline \multirow{4}{*}{ Rotinas } & \multicolumn{6}{|c|}{ Conceitos positivos } & \multicolumn{6}{|c|}{ Conceitos negativos } & \multirow{4}{*}{$\begin{array}{c}\text { Total de } \\
\text { Pontos } \\
\text { obtidos em } \\
\text { cada rotina }\end{array}$} & \multirow{4}{*}{$\begin{array}{c}\text { Grau de } \\
\text { Satisfação } \\
\text { demonstrado } \\
\text { em cada } \\
\text { rotina } \\
\text { (0 a } 5 \\
\text { pontos) }\end{array}$} \\
\hline & \multirow{2}{*}{\multicolumn{2}{|c|}{$\begin{array}{l}\text { Totalmente } \\
\text { satisfeito } \\
\text { Conceito } 5\end{array}$}} & \multirow{2}{*}{\multicolumn{2}{|c|}{$\begin{array}{c}\text { Muito } \\
\text { satisfeito } \\
\text { Conceito } 4\end{array}$}} & \multirow{2}{*}{\multicolumn{2}{|c|}{$\begin{array}{l}\text { Satisfeito } \\
\text { Conceito } 3\end{array}$}} & \multirow{2}{*}{\multicolumn{2}{|c|}{$\begin{array}{l}\text { Insatisfeito } \\
\text { Conceito } 2\end{array}$}} & \multirow{2}{*}{\multicolumn{2}{|c|}{$\begin{array}{c}\text { Muito } \\
\text { insatisfeito } \\
\text { Conceito } 1\end{array}$}} & \multirow{2}{*}{\multicolumn{2}{|c|}{$\begin{array}{l}\text { Totalmente } \\
\text { insatisfeito } \\
\text { Conceito } 0\end{array}$}} & & \\
\hline & & & & & & & & & & & & & & \\
\hline & $\mathrm{NL}$ & PT & $\mathrm{NL}$ & PT & NL & PT & $\mathrm{NL}$ & PT & $\mathrm{NL}$ & PT & $\mathrm{NL}$ & PT & & \\
\hline Nas rotinas de recepção & 7 & 35 & 2 & 8 & 0 & 0 & 0 & 0 & 0 & 0 & 0 & 0 & 43 & 4,78 \\
\hline $\begin{array}{l}\text { Nas rotinas dos setores } \\
\text { financeiros }\end{array}$ & 2 & 10 & 6 & 24 & 1 & 3 & 0 & 0 & 0 & 0 & 0 & 0 & 37 & 4,11 \\
\hline $\begin{array}{l}\text { Nas rotinas dos setores } \\
\text { técnicos }\end{array}$ & 5 & 25 & 3 & 12 & 1 & 3 & 0 & 0 & 0 & 0 & 0 & 0 & 40 & 4,44 \\
\hline $\begin{array}{l}\text { Nas rotinas de controle de } \\
\text { qualidade }\end{array}$ & 0 & 0 & 0 & 0 & 0 & 0 & 0 & 0 & 1 & 1 & 8 & 0 & 1 & 0,11 \\
\hline Nas atividades de compras & 0 & 0 & 0 & 0 & 0 & 0 & 0 & 0 & 3 & 3 & 6 & 0 & 3 & 0,33 \\
\hline $\begin{array}{l}\text { Nas atividades de controle } \\
\text { de estoque }\end{array}$ & 0 & 0 & 0 & 0 & 0 & 0 & 2 & 4 & 1 & 1 & 6 & 0 & 5 & 0,56 \\
\hline $\begin{array}{l}\text { Na emissão de relatórios } \\
\text { estatísticos }\end{array}$ & 0 & 0 & 0 & 0 & 4 & 12 & 2 & 4 & 2 & 2 & 1 & 0 & 18 & 2 \\
\hline $\begin{array}{l}\text { Na emissão de relatórios } \\
\text { gerenciais }\end{array}$ & 0 & 0 & 0 & 0 & 0 & 0 & 1 & 2 & 3 & 3 & 5 & 0 & 5 & 0,56 \\
\hline $\begin{array}{l}\text { No cálculo e na gestão de } \\
\text { custos de exames }\end{array}$ & 0 & 0 & 0 & 0 & 0 & 0 & 0 & 0 & 2 & 1 & 7 & 0 & 2 & 0,22 \\
\hline
\end{tabular}

$N L=$ número de laboratórios que selecionaram o conceito em questão; $P T=$ pontos obtidos no conceito em questão ( $N L$ x conceito); grau de satisfação = média ponderada dos conceitos aplicados (total de pontos obtidos em cada rotina/total de laboratórios participantes).

interno, ou os custos de operacionalização de amostras em caráter de urgência, entre outros.

Além dessa falta de subsídios suficientes para a incorporação da grande maioria dos custos envolvidos nas diferentes fases de produção, os sistemas em questão são deficientes na capacidade de fornecer dados para o controle de insumos, gerenciamento de perdas, controle de produtividade, avaliação da viabilidade econômica de determinadas metodologias ou equipamentos, entre outros. Os relatórios que disponibilizam são voltados à demonstração bastante simplista de rotinas e momentos operacionais, com pouco valor como ferramentas para o cálculo do custo real dos exames. Tal fato é ainda prejudicado pela dificuldade demonstrada por alguns dos entrevistados em perceber muitos dos custos indiretos envolvidos na execução dos exames, principalmente pelo fato de não serem profissionais atuantes diretamente nas áreas técnicas do laboratório que gerenciam.

Insatisfeitos com o desempenho do sistema para as atividades gerenciais e, mais do que nunca, necessitados de dados sustentáveis para consolidar atitudes, favorecer a tomada de decisões e subsidiar estratégias, os laboratórios estão buscando alternativas e soluções individuais para suprir a carência de ferramentas efetivas para tanto. Os gestores utilizam-se como podem das informações cedidas pelo LIS e as associam a técnicas pessoais de cálculo, visando obter resultados os mais próximos possíveis da realidade e que possam ser empregados na tomada de decisões acertadas. Além de pouca solidez, estas metodologias, baseadas mais no empirismo do que em dados reais, acabam determinando uma falta de homogeneidade por parte dos laboratórios, quando lançam mão dos custos assim calculados, por exemplo, para estabelecer o preço de venda do seu produto, tornando este bastante diversificado quando apresentado ao mercado.

Os colaboradores entrevistados que estiveram envolvidos no desenvolvimento do LIS afirmaram não ter conhecimentos específicos em muitas das atividades laboratoriais, o que por si só dificulta a implementação de melhorias condizentes com as necessidades citadas pelos gestores. Tem-se também que menos da metade desses laboratórios possui um departamento de informática próprio. Tais fatos demonstraram a necessidade de envolvimento de uma equipe multidisciplinar nos projetos de desenvolvimento e manutenção dos sistemas de informática laboratorial, cuja integração pode prover observações de suma importância para a compreensão do processo analítico como um todo e, assim, permitir o desenvolvimento de ferramentas mais adequadas aos estudos que se deseja fazer. 
O tempo em que o LIS encontra-se em operação nos laboratórios pesquisados variou de oito meses a 18 anos, diferença esta que não trouxe grandes variações nas observações pertinentes a este estudo. Os sistemas parecem não ter evoluído com o passar do tempo, tendo-se mudado muitas vezes as plataformas em que trabalham, mas permanecendo seu foco operacional somente. Não foi possível definir se a ausência de grandes diferenças entre eles quanto aos aspectos levantados deve-se à hipótese de serem sistemas que não permitem ou não comportam os recursos necessários ao fornecimento dos dados necessários ao cálculo e à gestão do custo dos exames, ou à idéia de que a necessidade de tais informações é por demais recente e que somente hoje esteja sendo levada em consideração com a devida intensidade.

\section{Conclusões}

O estudo confirmou a hipótese de que os sistemas de informática laboratorial atualmente disponíveis não oferecem os subsídios necessários ao suprimento das necessidades apresentadas pelos laboratórios de análises clínicas, tornando o cálculo do custo de seu produto principal, o resultado do exame, uma atividade individual, empírica e por demais manual em uma era em que a informatização reina quase absoluta. Os sistemas de informação implementados nos laboratórios pesquisados são tidos por seus usuários como satisfatórios para as atividades operacionais, base de sua formatação original, mas inadequados a atividades de cunho gerencial.

Sendo os sistemas de informática capazes de armazenar, gerenciar e integrar tantas informações, parece difícil compreender por que os laboratórios passam por tanta dificuldade para obter relatórios capazes de subsidiar cálculos de custos e decisões técnico-administrativas. Concluiu-se que, diante das particularidades do laboratório de análises clínicas, o desenvolvimento de sistemas destinados a tal finalidade pode requerer o envolvimento de uma equipe multidisciplinar que conheça a fundo a atividade laboratorial e os pontos relacionados à gestão de serviços de saúde, tendo em mente uma visão técnica sem perder, entretanto, o foco empresarial.

\section{Referências}

I. ALVES, S. L:; QUICUCO, O. Cálculo do resultado financeiro em laboratórios clínicos. Rio de Janeiro: Diagraphic, 2000.

2. BIZE, R. et al. El costo estándar: teoría y aplicación. Revista Chilena Salud Pública, v. 33, n. I, p. 15-21, 1999.

3. CHAE, Y. et al. Development of an intelligent laboratory information system for health promotion. Informs \& Korms, Seoul Korea, 2000.

4. FELDER, R. A. Automation: survival tools for the hospital laboratory. Second International Bayer Diagnostics Laboratory Testing Symposium. New York City, 1998.

5. FIEDMAN, B. A. The total laboratory solution: a new laboratory e-business model based on a vertical laboratory metanetwork. Clinical Chemistry, v. 47, n. 8, p. 1526-35, 2001.

6. MARKIN, R. et al. Laboratory automation: trajectory, technology and tacties. Clinical Chemistry, v. 46, n. 5, p. 764-7I, 2000.

7. MAYER, M. et al. Costo de la administración del laboratorio. Acta Bioquímica Clínica Latinoamericana, v. 3 I, n. 2, p. 217-27, 1997.

8. NARAYANAN, S. Techonology and laboratory instrumentation in the next decade. Medical Economics, v. 32, n. I, p. 24-31, 2000.

9. OGUSHI, Q.; ALVES, S. L. Administração em laboratórios clínicos. Rio de Janeiro: Atheneu, 1998.

10. OPPOLZER, E. M.; MULLER, M. M. Health care system and laboratory medicine in Austria: present status and future perspectives. Clinica Chimica Acta, v. 290, p. 37-55, 1999.
I I. PANSINO, N. The national health system: future possibilities for the clinical laboratory. Clinica Chimica Acta, v. 319, p. I0I-5, 2002

12. SANNAZZARO, C. A. Contribuição para o estudo dos custos unitários de análises bioquímicas quantitativas realizadas pelo processo manual e pelo processo automático no laboratório de análises clínicas do Hospital Universitário da Universidade de São Paulo em 1989. 1993. Tese (mestrado) - Universidade de São Paulo, São Paulo.

13. SARKOZI, L. et al.The effects of total laboratory automation on the management of a clinical chemistry laboratory. Clinica Chimica Acta, v. 329, n. 2, p. 89-94, 2003.

I4. SILVERSTEIN, J. C.; ROTHSCHILD,A. S. Clinical perspectives on the modern laboratory. Clinical Laboratory Medicine, v. 19 n. 2, p. $421-32,1999$

15. TRUCHAUD, A. et al. New tools for laboratory design and management. Clinical Chemistry, v. 43, n. 9, p. 1709-15, 1997.

16.YOUNG, D. S. et al. Laboratory costs in the context of disease. Clinical Chemistry, v. 46, n. 7, p. 967-75, 2000.

17. BLICK, KENNETH, E. Decision-making laboratory computer system as essential tools for achievement of total quality Clinical Chemistry, v. 43, n. 5, p. 908-12, 1997.
Endereço para correspondência

Katia Cristina Ugolini Mugnol Universidade de Mogi das Cruzes Centro Interdisciplinar de Investigação Bioquímica Avenida Dr. Cândido Xavier de Almeida e Souza, 200 CEP 08780-911 - Mogi das Cruzes-SP

Tel.: (11) 4798-7102/9895-0539 e-mail: kcum@uol.com.br 